44.4\% in $15 \mathrm{~Hz}$ group ( $\mathrm{p} \geq 0.480$ ). One patient had numbness/ tingling in the right arm in $5 \mathrm{~Hz}$ group.

Conclusions In this interim analysis, repetitive translumbar and transsacral magnetic stimulation appears safe, and at $1 \mathrm{~Hz}$ frequency showed significant superiority when compared to higher frequencies for the treatment of FI. This non-invasive neuromodulation modality offers promise as a novel treatment approach for FI.

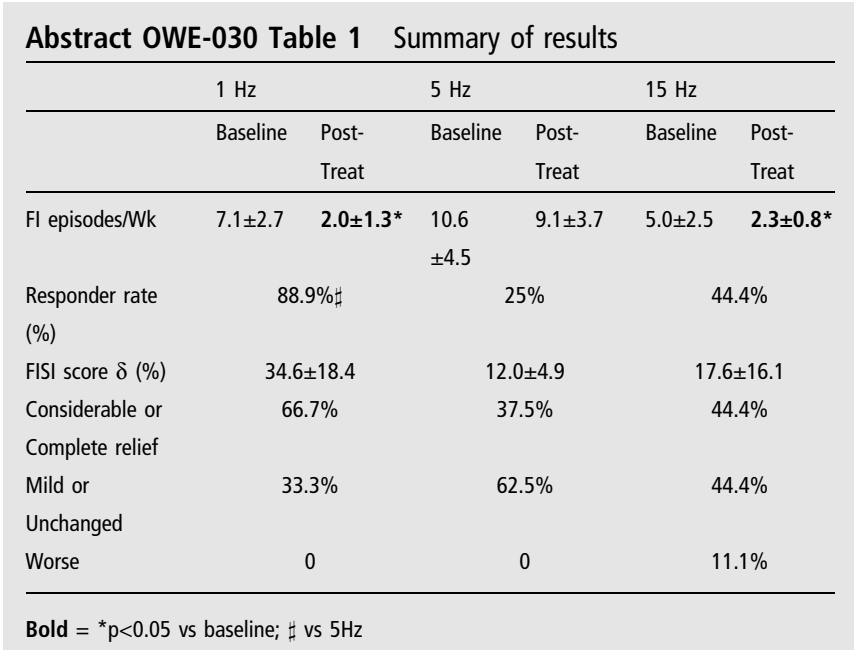

\section{OWE-031 OESOPHAGEAL APERISTALSIS IS UNDER INVESTIGATED IN THOSE WITHOUT ACHALASIA OR REFLUX}

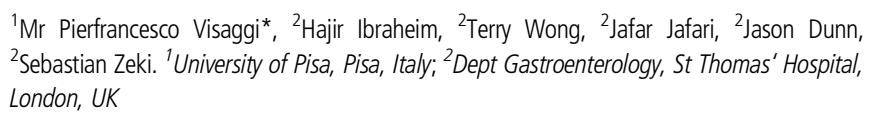

\subsection{6/gutjnl-2018-BSGAbstracts.419}

Introduction Oesophageal aperistalsis (OA) is the absence of oesophageal motility with water swallows at high-resolution manometry (HRM). The main causes are achalasia and reflux although in many patients no cause is found, therefore we aimed to investigate the number of patients with an identifiable cause of $\mathrm{OA}$ and the number of patients in whom the most common aetiologies have been determined. There is no consensus for the investigation of OA without achalasia; this will depend on how common the underlying aetiology is.

Methods We examined the reports of patients who had HRM at Guy's and St. Thomas' NHS Trust from January 2008 to July 2017. 492 patients had OA as per the Chicago Classification 2014; achalasia was defined as an integrated relaxation pressure (IRP) of $>15 \mathrm{mmHg}$ or IRP $12-15 \mathrm{mmHg}$ and a barium swallow or other imaging or a previous myotomy for achalasia was identified. For those without achalasia, Gastroesophageal reflux disease (GORD) was defined according to any $\mathrm{pH}$ study off PPI. Patients without GORD or achalasia were classified as non-achalasia, nonreflux aperistalsis (NANRA). Non-achalasia patients without a $\mathrm{pH}$ study were excluded $(n \geq 35)$. The electronic patient record of NANRA patients was consulted to look for evidence of autoimmune disorders (AD), eosinophilic oesophagitis (EoE) or previous oesophageal surgery.

Results Among 457 included patients we defined three categories: $183(40 \%)$ had achalasia, $185(41 \%)$ had GORD and 89 (19\%) had NANRA.
Of the 89 NANRA patients, 29\% had an AD including Systemic Lupus Erythematosus, Scleroderma, Sjögren syndrome and Antisynthetase syndrome $(\mathrm{n} \geq 25, \quad \mathrm{M}: \mathrm{F} \quad 3: 7$, average age $\geq 48)$. One had Myotonic Dystrophy $(n \geq 1) ; 11 \%(n \geq 10)$ had hypersensitive oesophagus; $6 \% \quad(n \geq 5)$ had surgery for atresia, oesophageal spasm, or gastric cancer; $2 \%(\mathrm{n} \geq 2)$ had EoE and in $2 \%(n \geq 2)$ of patients $A D$ screen and EoE screen were normal. The remaining $50 \%$ of NANRA patients $(n \geq 44)$ had an unknown cause but incomplete investigations (no screen for AD: 97.7\%; no biopsy: 67.4\%).

Conclusions 1.The principal cause of OA is achalasia; it shouldn't be dismissed as a cause even if the IRP is $<15 \mathrm{mmHg}$ as $6.5 \%(\mathrm{n} \geq 12)$ of patients with achalasia and OA had IRP $<15 \mathrm{mmHg}$ but typical radiological findings.

2.GORD is present in $41 \%$ of patients but it is unclear whether it is a cause or effect of OA, therefore the finding of GORD should not stop further investigation.

3.Patients with $\mathrm{OA}$ are under investigated for $\mathrm{AD}$ and EoE. $50 \%$ of patients with NANRA had incomplete investigations potentially losing the opportunity to identify other aetiologies. It is unclear whether NANRA patients should be routinely tested for $\mathrm{AD}$ or for $\mathrm{EoE}$, or whether this should be done only in selected cases.

\section{OWE-032 A RANDOMISED PLACEBO-CONTROLLED TRIAL OF A MULTI-STRAIN PROBIOTIC FORMULATION. (BIO-KULT ${ }^{\circledR}$ ) IN THE MANAGEMENT OF IBS-D}

Shamsuddin Ishaque*, Sheikh Mohammed Khosruzzaman, Dewan Saifuddin Ahmed, Mukesh Prasad Sah, Malwina Naghibi*. Bangabandhu Sheikh Mujib Medical University (BSMMU), Dhaka, Bangladesh

\subsection{6/gutjnl-2018-BSGAbstracts.420}

Introduction Increasing evidence supports the viewpoint that alterations in the diversity and function of gastrointestinal bacteria contributes to IBS, and that increasing the mass of beneficial species, by consuming probiotics, may lower pathogenic bacteria numbers and help alleviate symptoms.

Methods In this double-blind trial, a total of 360 adult patients with moderate-to-severe symptomatic diarrhoeapredominant IBS (IBS-D) were randomised to receive either treatment with the multi-strain probiotic Bio-Kult (14 different bacterial strains) or placebo for 16 weeks. The primary outcome measure was change in abdominal pain. The secondary outcomes included frequency of bowel motions, overall change in IBS-severity scoring system (IBS-SSS) and IBS specific quality of life (IBS-QoL).

Results In comparison to placebo, treatment with probiotics significantly alleviated the severity of abdominal pain in patients with IBS-D: 69\% reduction for probiotic versus $47 \%$ for placebo $(p<0.001)$, equating to a 145 point reduction on the IBS-SSS. The level of patients rating their symptoms as moderate-to-severe was reduced from $100 \%$ at baseline to $14 \%$ in the multi-strain probiotic group by follow-up (month $5)$ versus $48 \%$ for placebo $(p<0.001)$. In addition, the number of bowel motions per day from month 2 onwards was significantly reduced in the probiotic group compared with the placebo group $(\mathrm{p}<0.05)$. In addition to relieving symptoms, the probiotic markedly improved all dimensions of quality of life in the 34-item IBS-QoL questionnaire. No serious adverse events were reported.

Conclusions The multi-strain probiotic was associated with significant improvement in symptoms in IBS-D patients, and was well- 
tolerated. These results indicate that probiotic supplementation confers benefit in IBS-D and deserves further investigation.

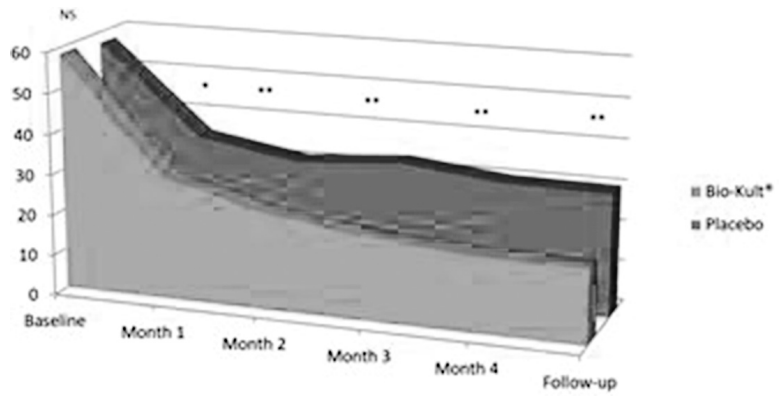

Abstract OWE-032 Figure 1 lbs-sss abdominal pain rating with probiotic (bio kult $(\mathrm{X})$ or placebo(16 weeks treatment and1-month follow up

The lower the score less the pain: $* \mathrm{p} \geq 0.002 * \mathrm{p} \leq 0.001$ : NS $\geq$ Not significant

\section{PWE-127 GASTROINTESTINAL DYSMOTILITY - THE CONUNDRUM OF FEEDING: A NEW TECHNIQUE}

Rebecca Anderson*, Simon Higgs, Steve Hornby, Richard Makins. Gloucestershire Hospitals NHS Foundation Trust, Cheltenham, UK

\subsection{6/gutjnl-2018-BSGAbstracts.421}

Introduction Gastrointestinal (GI) dysmotility disorders can be debilitating and their management challenging. Patients often require feeding tubes to support nutrition, which can be complicated by discomfort and/or displacement. Laparoscopically Assisted-Enteroscopically Positioned Jejunostomy Tube (LAEPJT) insertion is a novel technique not previously described in GI literature. It involves enteroscopic insertion of a jejunostomy tube under direct laparoscopic vision and general anaesthesia. This study reviews the experience of LA-EPJT insertion for GI dysmotility disorders at a district hospital.

Methods Endoscopy records were used to identify all patients who had undergone LA-EPJT insertion. Patient notes were reviewed to identify indications, previous tube requirements and outcomes.

Results 7 LA-EPJTs were placed between 2014 and 2017 (6 females; age 19-77; median 35; mean 43). Table 1 shows patient symptoms and indications for LA-EPJT insertion.

No tubes migrated or were dislodged after insertion. 1/7 patients $(14.3 \%)$ had early bleeding and tube-site infection. Perforation rates were $0 \%$. $5 / 7(71.4 \%)$ had initial pain with feeding after LA-EPJT insertion although this universally subsided, 1/7 (14.3\%) had ongoing vomiting. 5/7 (71.4\%) tubes remain in situ. 1 was replaced with a PEG-J to allow venting of intestinal contents, and 1 was removed and TPN commenced (psychological factors precluded continued tubefeeding).

Conclusions This study suggests that LA-EPJTs are useful nutritional adjuncts for patients with GI dysmotility disorders. They are a more permanent solution, with little risk of migration. They appear to better control symptoms such as pain and vomiting than traditional tubes, and are simple to change. The procedure benefits from direct laparoscopic vision of the jejunum and therefore carries low perforation rates.

Further studies are needed to assess long-term complications and efficacy of LA-EPJTs for symptom control and nutrition.
However, this data is promising and GI centres could consider adoption of this novel technique for patients with GI dysmotility disorders.

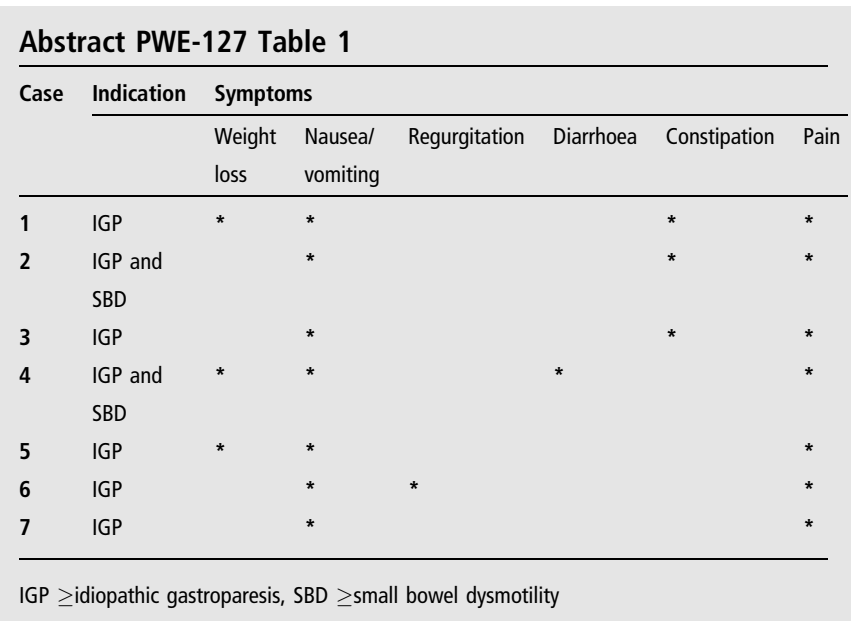

Previous non-pharmacological treatments and nutritional support utilised by patients are shown in table 2 .

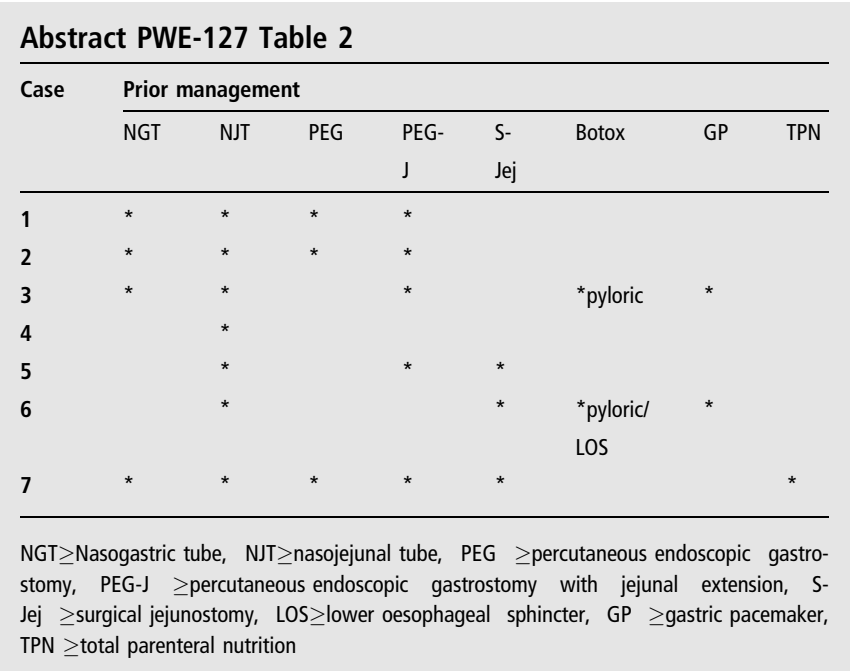

\section{PWE-128 HYPNOTHERAPY FOR IRRITABLE BOWEL SYNDROME: THE PATIENT'S PERCEPTION}

Anne-sophie Donnet*, Shariq Hasan, Vivien Miller, Peter Whorwell. Wythenshawe Hospital, Manchester, UK

\subsection{6/gutjnl-2018-BSGAbstracts.422}

Background Numerous studies have shown that hypnotherapy (HT) improves the symptoms of irritable bowel syndrome (IBS) using clinical outcome measures. In light of the increasing interest in capturing the patient's perception of their illness and treatment, it was felt it would be helpful to record how patients perceive the hypnotherapeutic process, on which there is currently little information.

Aims In addition to measuring symptom change, we have recently started to record the patient's perception of hypnotherapy for their IBS, including their expectations, and now report the results for the first 50 patients.

Methods 50 consecutive IBS patients (38 females and 12 males, age range 18-76) attending for hypnotherapy were 